\title{
Multiple Mutations of CYP2C19, PON1 and ABCB1 Influence Platelet Response, Bleeding and Thrombosis Risk to Clopidogrel after Percutaneous Coronary Intervention: A Retrospective Study
}

Hui Jin

The Fifth People's Hospital of Shanghai, Fudan University

Jinfei Song

The Fifth People's Hospital of Shanghai, Fudan University

Xiaoying Shen

The Fifth People's Hospital of Shanghai, Fudan University

Qing Liang

The Fifth People's Hospital of Shanghai, Fudan University

Guangchun Sun

The Fifth People's Hospital of Shanghai, Fudan University

Yan Yu ( $\nabla$ yanyu10@fudan.edu.cn )

The Fifth People's Hospital of Shanghai, Fudan University

\section{Research Article}

Keywords: Multiple mutations, clopidogrel, bleeding, thrombosis, platelet aggregation rate

Posted Date: June 16th, 2021

DOI: https://doi.org/10.21203/rs.3.rs-582961/v1

License: (c) (i) This work is licensed under a Creative Commons Attribution 4.0 International License. Read Full License 


\section{Abstract}

Background:Different combinations of multiple mutations of CYP2C19, PON1and ABCB1 for the efficiency and safety of Clopidogrel in patients undergoing percutaneous coronary intervention ( $\mathrm{PCl}$ ) remain unclear.

Methods: 263 Chinese Han patients receiving 75mg Clopidogrel and 100mg Aspirin every day for 12 months after $\mathrm{PCl}$ were enrolled in this study. ADP-induced platelet aggregation rates, thrombosis and bleeding risk are used to compare the Clopidogrel response among the combined genetic mutation.

Results: Our study demonstrated that multiple genetic mutations are very common in patients receiving Clopidogrel and Aspirin after PCI.Patients who have high ADP-induced platelet aggregation rate with more mutations of CYP2C19, PON1 and ABCB1. Patients with full mutations of CYP2C19, PON1 and ABCB1 have highest risk of thrombosis and lowest risk of bleeding in patients receiving clopidogrel and aspirin with one-year follow-up duration.

Conclusions:We summarized multiple genetic polymorphism influences platelet reactivity, bleeding and thrombosis risk to Clopidogrel after percutaneous coronary intervention.

\section{Background}

Percutaneous coronary intervention(PCl)is a common revascularization strategy to Coronary atherosclerotic heart disease.Post-procedural thrombotic events often happen in the implanted vascular stent.Dual-antiplatelet therapystrategies after PCl, with aspirin and Clopidogrel, is firstly recommended to reduce the thrombosis risk in European and American Guidelines[1].However, clopidogrel's active metabolite generation is unpredictable, which was influenced by the function of CYP2C19, PON1 and ABCB1. Cytochrome 4502C19 (CYP2C19) catalyzes the prodrug clopidogrel into 2-oxo-clopidogrel[2].The paraoxonase (PON-1) enzyme transforms 2-oxo-clopidogrel into its active form $[3,4]$. The gene ABCB1 encoding theP Glycol-protein (P-gp) regulatesthe clopidogrel absorption in systemic circulation[5, 6].Patients carriedCYP2C19*2, CYP2C19*3, PON-1Q192R or ABCB1 C3435T homozygous mutant genotypesexperiencedlow clopidogrel response and a high risk of adverse thromboticeventswhen receiving aspirin and Clopidogrel after $\mathrm{PCl}$. While the evidence for effects of alleles heterozygous mutations of CYP2C19, PON1 and ABCB1 in patients with acute coronary syndrome (ACS) or coronary artery disease (CAD) undergoing PClreceiving Clopidogrel is still controversial and inconclusive[3, 5, 7-10]. The efficiency and safety of Clopidogrel on carriers of multiple heterozygous mutationsof CYP2C19, PON1 and ABCB1 also remains unclear. This study aims to demonstrate the influence of Clopidogrel on Platelet response, Bleeding and Thrombosis Risk in patients undergoing PCI with multiple heterozygous mutation of CYP2C19, PON1 and ABCB1 gene.

\section{Methods}

\section{Study Participants}

263 Chinese Han patients receiving 75mg Clopidogrel and 100mg Aspirin every day for 12 monthsafterPCl from The Fifth People's Hospital of Shanghai were enrolled in this retrospective study between June2016 and March 2020. The inclusion criteria were: (1) patients with genotyping of the CYP2C19*2, CYP2C19*3, PON-1Q192R and ABCB1 C3435T with polymerase chain reaction(PCR); (2) patients with regular following up for 12 months; (3) 
patients with continuous drug prescription records; (4) patients who calculated the ADP dependent Platelet aggregation rate when clopidogrel treated for at least 7 days and stayed at a stable plasma concentration. The exclusion criteria: (1) patientswho experienced the major bleeding and thrombotic events in the last 6 months before PCl; (2)Patients who received anticoagulation drugs in the last 6 months before PCl; (3) patients received CYP2C19 or ABCB1 inhibitors (such as Omeprazole or Clarithromycin)during 12 months after PCl; (3) patients who stopped or adjusted the dose of clopidogrel and aspirin during dual-antiplatelet therapy; (4) patients who switched to ticagrelor or prasugrel;(5)patients with severerenal and hepatic function failure.(6)patients with large surgery during the observation period.

The study was approved by the ethics committee ofThe Fifth People's Hospital of Shanghai.

\section{Platelet aggregation assays}

Blood samples for platelet aggregation ratio assays were done after 7 days with clopidogrel and aspirin in patients undergoing $\mathrm{PCl}$. Blood samples $(3 \mathrm{ml})$ werecollected in the tube containing $10 \mu \mathrm{mol} / \mathrm{L}$ sodiumcitrate. The platelet-rich plasma (PRP) and the plateletpoor plasma (PPP) were separated by centrifuged. ThePPP and PRP samples were heated to $37^{\circ} \mathrm{C}$ for $3 \mathrm{~min}$,then $20 \mu \mathrm{mol} / \mathrm{L}$ of $A D P$ was added to the samples tomeasure platelet aggregation rate (PAR).

\section{Genotyping}

Details on Genotyping technology has been published previously[3,11]. All patients enrolled in this study have undergone genetic testing for CYP2C19, ABCB1 and PON1 genotypes. The DNA were extracted and the single nucleotide polymorphisms (SNPs) of each allele(CYP2C19*2, CYP2C19*3, ABCB1 and PON-1) were determined by the DNA assay method on a Real-Time PCR System according to the manufacturer's instructions. Carrier status for all alleles was available and reported as wild type, heterozygous or homozygous for the minor allele.

\section{Outcomes and follow-up}

The thrombotic and bleeding events were extracted from out-patients follow-ups for 12 months after PCI. Thrombotic eventsare composed of stent thrombus,Stroke, TIA and target vessel-related MI. The bleeding events is consisting of major bleeding (Cerebral hemorrhage, Gastrointestinal bleeding, Fundus bleeding) and minor bleeding (Subcutaneous bleeding, Gums bleeding and so on)

\section{Statistical analysis}

Variables are presented as the mean \pm standard deviation (SD) or frequencies (percentage). Continuous variables were compared using Student's t-test or one-way ANOVA, and categorical variables were compared using the chisquared test or Fisher's exact test. To examine the association between the risk factors and the ADP induced platelet aggregation rate, we performed univariate and multivariate Cox regression analyses. To avoid the potential bias of covariates between high and low ADP induced platelet aggregation rate, a casecontrol matching method was used to match variables that included age, gender, hemoglobin and hepatic function. Matching tolerance was 0.02 . To compare the predictability for high ADP induced platelet aggregation rate among the genotyping mutation, logistic regression analysis was performed. Thrombosis and bleeding event rates were estimated by the Kaplan Meier method according to the multiple genetic mutation groups and compared by logrank tests. 


\section{Results}

\section{Identification of Multiple mutations of CYP2C19, ABCB1 and PON-1 in Han Chinese Partients.}

CYP2C19, PON-1 and ABCB1 genotypes were assayed in 263patients undergoing PCl. The distribution of CYP2C19*2, CYP2C19*3,PON Q192R and ABCB1 $3435 \mathrm{C}$ allelic and genotype frequency are shown in Table 1.Approximately $46 \%$ of the population had one or two heterozygous mutation of CYP2C19* 2 or CYP2C19*3 (CYP2C19 Intermediate metabolite, IM) and 14\% had homozygous alleles (CYP2C19 poor metabolite, PM). In terms of the prevalence of ABCB1 $3435 \mathrm{C}>\mathrm{T}$ polymorphism, $40 \%$ of the population had one T-allele (CT, heterozygous mutation) and $17 \%$ had two T-alleles (TT, homozygous mutation). In terms of the prevalence of PON1 polymorphism, $46 \%$ of the population had one or more A-allele (GA or AA) and 18\% had two A-alleles (AA). Only19 patients with no mutation of CYP2C19, PON1 and ABCB1 gene; 24 patients with three heterozygous mutation of CYP2C19, PON1 and ABCB1 gene; 25 patients with single homozygous mutation alone in CYP2C19, PON1 and ABCB1 gene (Table 1). 
Table 1

The distribution of multiple mutations of CYP2C19, ABCB1 and PON-1

\begin{tabular}{|c|c|c|c|c|}
\hline & \multirow[t]{2}{*}{ Total } & \multicolumn{3}{|c|}{ CYP2C19 metabolic phenotype } \\
\hline & & $\mathrm{EM}(* 1 / * 1)$ & $\operatorname{IM}(* 1 / * 2, * 3)$ & $\mathrm{PM}(* 2 / * 2)$ \\
\hline CYP2C19 metabolic phenotype & 263 & $97(0.37)$ & $126(0.48)$ & $40(0.15)$ \\
\hline \multicolumn{5}{|l|}{ ABCB1 3435C > T genotype } \\
\hline $\mathrm{CC}$ & $107(0.41)$ & $31(0.32)$ & $60(0.48)$ & $16(0.4)$ \\
\hline TC & $109(0.41)$ & $41(0.42)$ & $47(0.37)$ & $21(0.53)$ \\
\hline TT & $47(0.18)$ & $25(0.26)$ & $19(0.15)$ & $3(0.08)$ \\
\hline \multicolumn{5}{|l|}{ PON1 575A > G genotype } \\
\hline GG & $92(0.35)$ & $29(0.3)$ & $46(0.37)$ & $17(0.43)$ \\
\hline GA & $127(0.48)$ & $46(0.47)$ & $62(0.49)$ & $19(0.48)$ \\
\hline AA & $44(0.17)$ & $22(0.23)$ & $18(0.14)$ & $4(0.10)$ \\
\hline \multicolumn{5}{|c|}{ Combined genetic status(ABCB1 + PON1) } \\
\hline 0 mutation & $36(0.14)$ & $9(0.09)$ & $21(0.17)$ & $6(0.15)$ \\
\hline \multicolumn{4}{|l|}{ (ABCB1 CT or PON1 GA) } & $21(0.53)$ \\
\hline $\begin{array}{l}\text { ABCB1 }(\mathrm{mu})+\text { PON1(mu) } \\
(\mathrm{ABCB} 1 \mathrm{CT}+\mathrm{PON} 1 \mathrm{GA})\end{array}$ & $100(0.38)$ & $46(0.47)$ & $41(0.33)$ & $13(0.33)$ \\
\hline \multicolumn{5}{|c|}{ The values are expressed as $n(\%)$ of patients. } \\
\hline \multicolumn{5}{|c|}{ 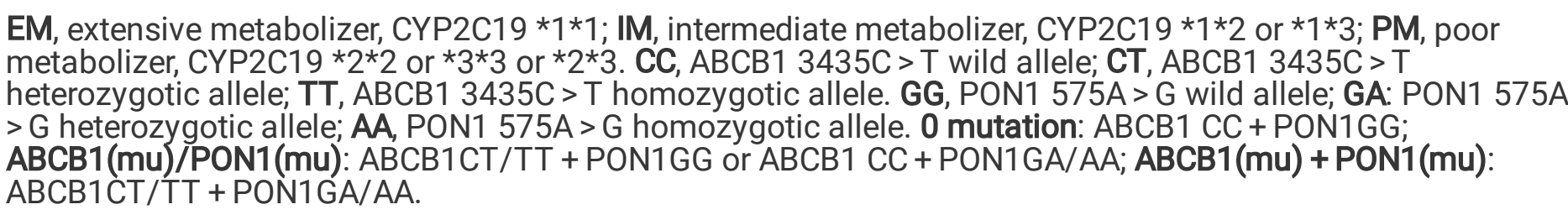 } \\
\hline
\end{tabular}

\section{Risk factorsof high platelet aggregation rate on patients with dual antiplatelet therapy.}

A total of 263 patients were enrolled in our study. Table 2 shows the baseline characteristics of the overall population according to the ADP dependent Platelet aggregation rate. The patients with high ADP-Platelet aggregation were older and had lowerhemoglobin. They also exhibited a higher prevalence of acute MI as an index diagnosis, chronic liver disease, CYP2C19 variants, as well as a greater incidence of higher ADP dependent Platelet aggregation compared with those who achieved a successful anti-platelet therapy (Table 2). 
Table 2

Patient characteristics of high and low ADP-induced Platelet aggregation

\begin{tabular}{|c|c|c|c|}
\hline \multirow[b]{2}{*}{ Covariste } & \multicolumn{2}{|c|}{ ADP-dependent Platelet Aggregation Rate } & \multirow{3}{*}{$P$ value } \\
\hline & $<50 \%$ & $\geq 50 \%$ & \\
\hline & $(n=157)$ & $(n=60)$ & \\
\hline Age, y & $66.49 \pm 11.07$ & $71.53 \pm 9.55$ & 0.002 \\
\hline Male/Female & $130 / 27$ & $38 / 22$ & 0.002 \\
\hline BMI & $24.85 \pm 2.87$ & $24.26 \pm 2.86$ & 0.220 \\
\hline \multicolumn{4}{|l|}{ Medical history } \\
\hline Hypertension & $103(0.66)$ & $37(0.62)$ & 0.558 \\
\hline Diabetes & $50(0.32)$ & $23(0.38)$ & 0.366 \\
\hline AMI & $62(0.39)$ & $14(0.23)$ & 0.026 \\
\hline ACS & $24(0.15)$ & $11(0.18)$ & 0.585 \\
\hline Ischemic stroke & $11(0.07)$ & $3(0.05)$ & 0.591 \\
\hline $\mathrm{DBP}(\mathrm{mmHg})$ & $76.98 \pm 13.81$ & $74.48 \pm 11.56$ & 0.215 \\
\hline $\mathrm{SBP}(\mathrm{mmHg})$ & $132.87 \pm 22.64$ & $130.58 \pm 22.27$ & 0.504 \\
\hline \multicolumn{4}{|l|}{$\mathrm{mmHg})$} \\
\hline $\mathrm{HR}(/ \min )$ & $74.54 \pm 15.95$ & $73.78 \pm 12.52$ & 0.742 \\
\hline $\operatorname{PLT}\left(\times 10^{\wedge} 9 / \mathrm{L}\right)$ & $205.05 \pm 62.35$ & $198.92 \pm 69.45$ & 0.386 \\
\hline $\mathrm{HGB}(\mathrm{g} / \mathrm{L})$ & $138.15 \pm 16.38$ & $131.46 \pm 15.51$ & 0.007 \\
\hline $\mathrm{HbA}_{1 \mathrm{C}}(\%)$ & $6.54 \pm 1.48$ & $6.74 \pm 1.74$ & 0.384 \\
\hline Viscosity & $1.38 \pm 0.11$ & $1.37 \pm 0.12$ & 0.681 \\
\hline $\operatorname{ALT}(\mathrm{U} / \mathrm{L})$ & $31.16 \pm 28.04$ & $26.77 \pm 31.60$ & 0.321 \\
\hline $\mathrm{AST}(\mathrm{U} / \mathrm{L})$ & $83.82 \pm 120.44$ & $30.74 \pm 40.49$ & 0.001 \\
\hline $\mathrm{SCr}(\mu \mathrm{mol} / \mathrm{L})$ & $83.01 \pm 37.28$ & $84.20 \pm 25.48$ & 0.820 \\
\hline
\end{tabular}

The values are expressed as the mean \pm SD or $n(\%)$ of patients.

BMI, body mass index; AMI, acute myocardial Infarction; ACS, acute coronary syndromes; DBP, Diastolic blood pressure; SBP, Systolic blood pressure; HR: heart rate; HGB, hemoglobin : PLT, platelet count; $\mathrm{HbA}_{1 \mathrm{C}}$, hemoglobin A1c; Viscosity, blood viscosity; ALT, alanine aminotransferase; AST, Aspartate aminotransferase; SCr, Serum creatinine; TC, total cholesterol; TG, Triglycerides; eGFR, estimated glomerular filtration rate; CYP2C19 $1 * 1$, CYP2C19 wild allele; CYP2C19*1*2 or *1*3, CYP2C19 heterozygotic allele; CYP2C19 $2 * 2$ or *3*3 or $* 2 * 3$, CYP2C19 homozygotic allele. CC, ABCB1 3435C > T wild allele; CT, ABCB1 3435C > T heterozygotic allele; TT, ABCB1 3435C > T homozygotic allele. GG, PON1 575A>G wild allele; GA: PON1 575A $>$ G heterozygotic allele; AA, PON1 575A > G homozygotic allele. 


\begin{tabular}{|c|c|c|c|}
\hline \multicolumn{4}{|c|}{ ADP-dependent Platelet Aggregation Rate } \\
\hline $\mathrm{TC}(\mathrm{mmol} / \mathrm{L})$ & $3.83 \pm 1.07$ & $3.66 \pm 0.98$ & 0.281 \\
\hline TG (mmol/L) & $1.66 \pm 1.21$ & $1.48 \pm 0.80$ & 0.290 \\
\hline $\operatorname{eGFR}\left(\mathrm{ml} / \mathrm{min} / 1.73 \mathrm{~m}^{2}\right)$ & $82.27 \pm 20.29$ & $77.90 \pm 16.68$ & 0.175 \\
\hline CYP2C19 & & & 0.004 \\
\hline$* 1 * 1$ & $59(0.38)$ & $15(0.25)$ & 0.08 \\
\hline$* 1 * 2 / * 1 * 3$ & $75(0.48)$ & $33(0.55)$ & 0.341 \\
\hline$\star 2 * 2 / * 3 * 3$ & $23(0.15)$ & $12(0.2)$ & 0.338 \\
\hline \multicolumn{4}{|l|}{ PON1 } \\
\hline GG & $54(0.34)$ & $20(0.33)$ & 0.883 \\
\hline GA & $80(0.51)$ & $27(0.45)$ & 0.433 \\
\hline AA & $23(0.15)$ & $13(0.22)$ & 0.214 \\
\hline \multicolumn{4}{|l|}{ ABCB1 } \\
\hline $\mathrm{CC}$ & $67(0.43)$ & $25(0.42)$ & 0.302 \\
\hline CT & $63(0.4)$ & $21(0.35)$ & 0.448 \\
\hline TT & $27(0.17)$ & $14(0.23)$ & 0.707 \\
\hline \multicolumn{4}{|c|}{ The values are expressed as the mean \pm SD or $n(\%)$ of patients. } \\
\hline \multicolumn{4}{|c|}{ 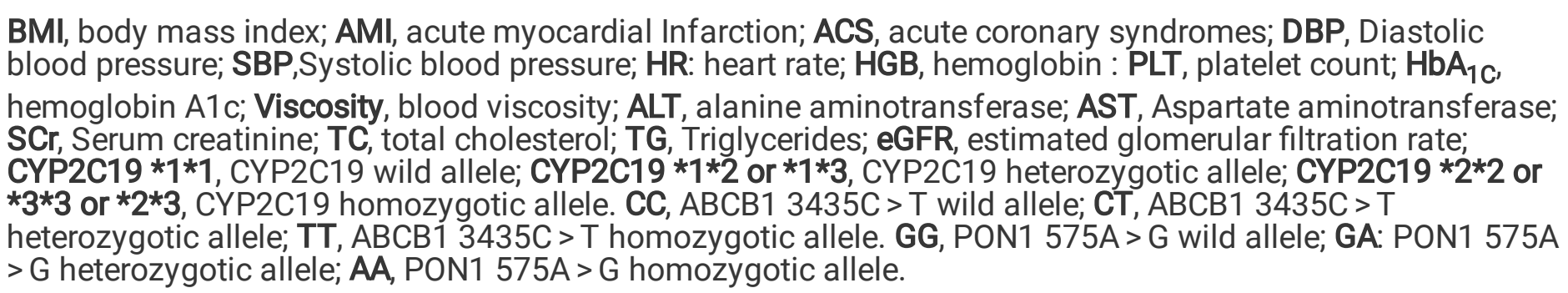 } \\
\hline
\end{tabular}

\section{Genetic polymorphism is associated with high platelet aggregation rate.}

To order to compare the difference distribution of genotyping mutation between high and low ADP-induced platelet aggregation rates, a 1:1 case-control matching procedure was performed to avoid the potential bias of covariates. After matching for age gender, $\mathrm{AMI}$, hemoglobin and hepatic function, there were no differences in age gender, $\mathrm{AMI}$, hemoglobin and hepatic functionafter matching between high and low ADP-induced platelet aggregation rates. There remained a significantly higher mutation of CYP2C19, PON1 and ABCB1 gene in high ADP-induced platelet aggregation rate (Table 3). 
Table 3

Relationship between genotype mutations and ADP-induced platelet aggregation rate after 1:1 propensity score matching.

\begin{tabular}{|c|c|c|c|c|}
\hline \multirow[b]{2}{*}{ Covariste } & \multicolumn{3}{|c|}{ ADP-dependent Platelet aggregation } & \multirow[b]{2}{*}{$\begin{array}{l}\text { Multivariate analysis } \\
\text { HR }(95 \% \mathrm{Cl})\end{array}$} \\
\hline & $\begin{array}{l}<50 \% \\
(n=47)\end{array}$ & $\begin{array}{l}\geq 50 \% \\
(n=60)\end{array}$ & $P$ value & \\
\hline Age, y & $68.42 \pm 7.46$ & $71.53 \pm 9.55$ & 0.478 & $0.970(0.890-1.506)$ \\
\hline Male/Female & $30 / 17$ & $38 / 22$ & 0.625 & $1.440(0.334-6.209)$ \\
\hline \multicolumn{5}{|l|}{ Medical history } \\
\hline Hypertension & $30(0.64)$ & $37(0.62)$ & 0.864 & $0.903(0.281-2.899)$ \\
\hline Diabetes & $12(0.26)$ & $23(0.38)$ & 0.944 & $0.944(0.192-4.647)$ \\
\hline AMI & $7(0.15)$ & $14(0.23)$ & 0.099 & $0.214(0.034-1.338)$ \\
\hline $\mathrm{HGB}(\mathrm{g} / \mathrm{L})$ & $132.28 \pm 14.04$ & $131.46 \pm 15.51$ & 0.459 & $1.019(0.969-1.073)$ \\
\hline $\mathrm{HbA}_{1 \mathrm{C}}(\%)$ & $6.54 \pm 1.61$ & $6.74 \pm 1.74$ & 0.670 & $1.089(0.735-1.613)$ \\
\hline $\operatorname{ALT}(U / L)$ & $20.95 \pm 12.49$ & $26.77 \pm 31.60$ & 0.100 & $1.045(0.992-1.101)$ \\
\hline AST (U/L) & $32.01 \pm 39.60$ & $30.74 \pm 40.49$ & 0.365 & $0.991(0.971-1.011)$ \\
\hline $\mathrm{SCr}(\mu \mathrm{mol} / \mathrm{L})$ & $78.36 \pm 28.08$ & $84.20 \pm 25.48$ & 0.665 & $1.006(0.980-1.032)$ \\
\hline $\mathrm{TC}(\mathrm{mmol} / \mathrm{L})$ & $3.89 \pm 1.06$ & $3.66 \pm 0.98$ & 0.133 & $0.571(0.274-1.187)$ \\
\hline TG (mmol/L) & $1.62 \pm 1.36$ & $1.48 \pm 0.80$ & 0.929 & $0.979(0.608-1.574)$ \\
\hline CYP2C19 & & & 0.001 & \\
\hline *1*1 & $24(0.51)$ & $15(0.25)$ & 1 & \\
\hline$\star 1 * 2 / * 1 * 3$ & $22(0.47)$ & $33(0.55)$ & $<0.001$ & $0.005(0.000-0.079)$ \\
\hline$\star 2 * 2 / * 3 * 3$ & $1(0.02)$ & $12(0.20)$ & 0.015 & $0.023(0.002-0.309)$ \\
\hline PON1 & & & 0.003 & \\
\hline GG & $24(0.51)$ & $20(0.33)$ & 1 & \\
\hline GA & $21(0.45)$ & $27(0.45)$ & 0.001 & $0.015(0.001-0.182)$ \\
\hline$A A$ & $2(0.04)$ & $13(0.22)$ & 0.015 & $0.055(0.005-0.565)$ \\
\hline ABCB1 & & & 0.025 & \\
\hline $\mathrm{CC}$ & $27(0.57)$ & $25(0.42)$ & 1 & \\
\hline
\end{tabular}

The values are expressed as the mean \pm SD or $n(\%)$ of patients.

AMI, acute myocardial Infarction; HGB, hemoglobin; $\mathrm{HbA}_{1 \mathrm{C}}$, hemoglobin $\mathrm{A}_{1 \mathrm{C}}$; $\mathrm{ALT}$, alanine aminotransferase; AST, Aspartate aminotransferase; SCr, Serum creatinine; TC, total cholesterol; TG, Triglycerides. 


$\begin{array}{lllll}\text { CT } & 17(0.36) & 21(0.35) & 0.007 & 0.74(0.011-0.493) \\ \text { TT } & 3(0.06) & 14(0.23) & 0.080 & 0.183(0.027-1.222)\end{array}$

The values are expressed as the mean \pm SD or $n(\%)$ of patients.

AMI, acute myocardial Infarction; $\mathrm{HGB}$, hemoglobin; $\mathrm{HbA}_{1 \mathrm{C}}$, hemoglobin $\mathrm{A}_{1 \mathrm{C}}$; ALT, alanine aminotransferase; AST, Aspartate aminotransferase; SCr, Serum creatinine; TC, total cholesterol; TG, Triglycerides.

\section{Multiple genetic mutations influenced the adverse outcomes of dual antiplatelet therapy.}

Subsequently, the bleeding and thrombotic events were analyzed to explore the correlation between multiple mutations and adverse outcomes. The rate of any bleeding and major thrombosis is significantly different in individual CYP2C19, PON1 and ABCB1 loss-of-function allele carriers compared with noncarriers. The lower risk of bleeding and higher risk of thrombosis was observed on interaction amongcarriers with different number of variants. Besides, regardless of carrying or not carrying CYP2C19 variants, PON1 or ABCB1 variants increased the rate of thrombotic events(Table 4). 
Table 4

The correlation between multiple mutations and adverse outcomes

\begin{tabular}{|c|c|c|c|c|c|c|c|c|}
\hline & \multirow{3}{*}{$\begin{array}{l}\text { Total } \\
\text { (263) } \\
\text { NO }\end{array}$} & \multicolumn{7}{|c|}{ adverse outcomes } \\
\hline & & \multicolumn{4}{|c|}{ Bleeding events } & \multicolumn{3}{|c|}{ Thrombotic events } \\
\hline & & Yes & $P$ value & & NO & Yes & $P$ value & \\
\hline Total & (215) & (48) & & & (234) & (29) & & \\
\hline \multicolumn{4}{|c|}{ CYP2C19 metabolic phenotype } & 0.169 & & & & 0.546 \\
\hline$\star 1 * 1$ & 97 & $73(0.34)$ & $24(0.5)$ & 0.037 & & $89(0.38)$ & $8(0.28)$ & 0.271 \\
\hline$\star 1 * 2 / * 1 * 3$ & 126 & $106(0.49)$ & $20(0.42)$ & 0.338 & & $110(0.47)$ & $16(0.55)$ & 0.406 \\
\hline$\star 2 \star 2 / * 3 * 3$ & 40 & $36(0.17)$ & $4(0.08)$ & 0.142 & & $35(0.15)$ & $5(0.17)$ & 0.747 \\
\hline \multicolumn{4}{|l|}{ ABCB1 3435C > T genotype } & 0.083 & & & & 0.006 \\
\hline $\mathrm{CC}$ & 107 & $94(0.44)$ & $13(0.27)$ & 0.034 & & $99(0.42)$ & $8(0.28)$ & 0.128 \\
\hline TC & 109 & $83(0.39)$ & $26(0.54)$ & 0.048 & & $99(0.42)$ & $10(0.34)$ & 0.420 \\
\hline TT & 47 & $38(0.18)$ & $9(0.19)$ & 0.86 & & $36(0.15)$ & $11(0.38)$ & 0.003 \\
\hline \multicolumn{4}{|l|}{ PON1 575A > G genotype } & 0.665 & & & & 0.006 \\
\hline GG & 92 & $77(0.36)$ & $15(0.31)$ & 0.549 & & $88(0.38)$ & $4(0.14)$ & 0.011 \\
\hline GA & 127 & $101(0.47)$ & $26(0.54)$ & 0.367 & & $112(0.48)$ & $15(0.52)$ & 0.695 \\
\hline AA & 44 & $37(0.17)$ & $7(0.15)$ & 0.659 & & $34(0.15)$ & $10(0.34)$ & 0.007 \\
\hline \multicolumn{8}{|c|}{ Combined genetic status(CYP2C19+ABCB1 + PON1) 0.021} & 0.036 \\
\hline $\begin{array}{l}\text { CYP2C19(No) + } \\
\text { ABCB1(No) }+ \\
\text { PON1(No) }\end{array}$ & 9 & $5(0.02)$ & $4(0.08)$ & & & $9(0.04)$ & $0(0)$ & \\
\hline $\begin{array}{l}\text { CYP2C19(No) }+ \\
\text { ABCB1 }(\mathrm{Mu}) / \mathrm{PON} 1(\mathrm{Mu})\end{array}$ & 42 & $35(0.16)$ & $7(0.15)$ & & & $41(0.18)$ & $1(0.03)$ & \\
\hline $\begin{array}{l}\text { CYP2C19(No) + } \\
\text { ABCB1(Mu) + } \\
\text { PON1(Mu) }\end{array}$ & 46 & $33(0.15)$ & $13(0.27)$ & & & $39(0.17)$ & $7(0.24)$ & \\
\hline $\begin{array}{l}\text { CYP2C19(Mu) + } \\
\text { ABCB1(No) }+ \\
\text { PON1(No) }\end{array}$ & 27 & $26(0.12)$ & $1(0.02)$ & & & $27(0.12)$ & $0(0)$ & \\
\hline
\end{tabular}

The values are expressed as $n(\%)$ of patients.

CC, ABCB1 3435C > T wild allele; CT, ABCB1 3435C > T heterozygotic allele; TT, ABCB1 3435C > T homozygotic allele. GG, PON1 575A > G wild allele; GA: PON1 575A > G heterozygotic allele; AA PON1 575A > $\mathrm{G}$ homozygotic allele. CYP2C19(No), CYP2C19*1*1; CYP2C19(Mu), CYP2C19*1*2 or *1*3 or *2*2 or *3*3; ABCB1(No), ABCB1 CC, ABCB1 3435C > T wild allele; ABCB1(Mu), ABCB1CT/TT, ABCB1 3435C > T heterozygotic or homozygotic allele; PON1(No), PON1GG, PON1 575A>G wild allele; PON1(Mu), PON1GA/AA, PON1 575A > G heterozygotic or homozygotic allele. 


\begin{tabular}{|c|c|c|c|c|c|}
\hline & Total & adverse 0 & comes & & \\
\hline & (263) & Bleeding & ents & Thrombo & events \\
\hline $\begin{array}{l}\text { CYP2C19(Mu) + } \\
\text { ABCB1(Mu)/PON1(Mu) }\end{array}$ & 85 & $74(0.34)$ & $11(0.23)$ & $74(0.32)$ & 11(0.38) \\
\hline $\begin{array}{l}\text { CYP2C19(Mu) + ABCB1 } \\
(\mathrm{Mu})+\text { PON1(Mu) }\end{array}$ & 54 & $42(0.2)$ & $12(0.25)$ & $44(0.19)$ & $10(0.34)$ \\
\hline The values are expresse & as $\mathrm{n}(\%$ & of patients & & & \\
\hline $\begin{array}{l}\text { CC, ABCB1 } 3435 \mathrm{C}>\mathrm{T} \text { w } \\
\text { homozygotic allele. GG, } \\
\text { G homozygotic allele. C } \\
\text { ABCB1(No), ABCB1 CC, } \\
\text { heterozygotic or homoz } \\
\text { PON1 575A > G heterozy }\end{array}$ & $\begin{array}{l}\text { allele; } \\
\text { ON1 } 57 \\
\text { 2C19( } \\
\text { 3CB1 3 } \\
\text { otic alle } \\
\text { otic or h }\end{array}$ & $\begin{array}{l}\text { T, ABCB13 } \\
\text { A }>\text { G wild } \\
\text { o), CYP2C1 } \\
\text { 35C > T wilc } \\
\text { e; PON1(N } \\
\text { mozygotic }\end{array}$ & $\begin{array}{l}35 \mathrm{C}>\text { T hete } \\
\text { ele; GA: PON } \\
1 * 1 ; \text { CYP2C } \\
\text { Ilele; ABCB } \\
\text { PON1GG, P } \\
\text { llele. }\end{array}$ & $\begin{array}{l}\text { T, ABCB1 } 3 \\
\text { zygotic alle } \\
\star 1 * 2 \text { or } * 1 * \\
\text { TT, ABCB13 } \\
\text { t allele; POr }\end{array}$ & $\begin{array}{l}5 C>T \\
\text { AA, PON1 } 575 A> \\
\text { r*2*2 or *3*3; } \\
35 C>\text { T } \\
(\mathrm{Mu}), \text { PON1GA/AA, }\end{array}$ \\
\hline
\end{tabular}

\section{Difference between multiple heterozygous mutation and single homozygous mutation}

In order to compare the difference between multiple heterozygous mutation and single homozygous mutation, we extracted the information of patients with three heterozygous and single homozygous mutation of CYP2C19, PON1 and ABCB1 polymorphism. Patients with multiple heterozygous genotype in the clopidogrel plus aspirin group had a higher risk of bleeding ( $33 \%$ vs $8 \% ; P=0.028$ for Chi-square test) and lower risk of thrombosis ( $0 \%$ vs $24 \% ; p=0.01$ for Chi-square test) (Table 5). While,the thrombosis risk are not different in the two groups. 
Table 5

Baseline Characteristics Between Single Homozygous mutation and multiple Heterozygous mutation in Patients with Clopidogrel Treatment After PCl

\begin{tabular}{|c|c|c|c|}
\hline \multicolumn{4}{|c|}{ Genotyping mutation } \\
\hline \multirow[t]{3}{*}{ Covariste } & 3 Heterozygous & 1Homozygous & $P$ value \\
\hline & 0 Homozygous & 0 Heterozygous & \\
\hline & $(n=24)$ & $(\mathrm{n}=25)$ & \\
\hline Age, y & $68.96 \pm 14.08$ & $66.44 \pm 11.14$ & 0.490 \\
\hline Male/Female & $17 / 7$ & $18 / 7$ & 0.928 \\
\hline BMI & $26.02 \pm 3.17$ & $24.82 \pm 2.6$ & 0.178 \\
\hline \multicolumn{4}{|l|}{ Medical history } \\
\hline Hypertension & $15(0.63)$ & $15(0.6)$ & 0.858 \\
\hline Diabetes & $11(0.46)$ & $7(0.28)$ & 0.196 \\
\hline AMI & $10(0.42)$ & $8(0.32)$ & 0.483 \\
\hline ACS & $4(0.17)$ & $5(0.2)$ & 0.763 \\
\hline Stroke & $1(0.04)$ & $3(0.12)$ & 0.317 \\
\hline SBP $(\mathrm{mmHg})$ & $132 \pm 23.18$ & $137.04 \pm 22.54$ & 0.444 \\
\hline PLT $\left(\times 10^{\wedge} 9 / \mathrm{L}\right)$ & $213.88 \pm 70.13$ & $197.75 \pm 54.05$ & 0.377 \\
\hline $\mathrm{HGB}(\mathrm{g} / \mathrm{L})$ & $131.5 \pm 24$ & $134.75 \pm 14.27$ & 0.571 \\
\hline ADP-PAR (\%) & $37.83 \pm 20.38$ & $39 \pm 21.88$ & 0.868 \\
\hline $\mathrm{HbA}_{1 \mathrm{c}}(\%)$ & $6.86 \pm 1.95$ & $6.99 \pm 1.93$ & 0.817 \\
\hline Viscosity & $1.39 \pm 0.12$ & $1.38 \pm 0.11$ & 0.846 \\
\hline $\operatorname{ALT}(U / L)$ & $30.07 \pm 15.27$ & $34.28 \pm 49.21$ & 0.690 \\
\hline AST (U/L) & $89.95 \pm 100.62$ & $59.44 \pm 91.33$ & 0.272 \\
\hline $\mathrm{SCr}(\mu \mathrm{mol} / \mathrm{L})$ & $78.96 \pm 18.59$ & $83.44 \pm 26.52$ & 0.505 \\
\hline $\mathrm{TC}(\mathrm{mmol} / \mathrm{L})$ & $3.93 \pm 1.01$ & $4.13 \pm 1.11$ & 0.521 \\
\hline TG (mmol/L) & $1.61 \pm 0.99$ & $1.79 \pm 1.05$ & 0.557 \\
\hline $\mathrm{eGFR}\left(\mathrm{ml} / \mathrm{min} / 1.73 \mathrm{~m}^{2}\right)$ & $82.9 \pm 17.37$ & $80.52 \pm 19.66$ & 0.680 \\
\hline
\end{tabular}

The values are expressed as the mean \pm SD or $n(\%)$ of patients.

BMI, body mass index; AMI, acute myocardial Infarction; ACS, acute coronary syndromes; SBP,Systolic blood pressure; HR: heart rate; HGB, hemoglobin : PLT, platelet count; ADP-PAR: ADP induced platelet aggregation rate; $\mathrm{HbA}_{1 \mathrm{C}}$, hemoglobin A1c; Viscosity, blood viscosity; ALT, alanine aminotransferase; AST, Aspartate aminotransferase; SCr, Serum creatinine; TC, total cholesterol; TG, Triglycerides; eGFR, estimated glomerular filtration rate. 


\section{Genotyping mutation}

$\begin{array}{llll}\text { Bleeding events } & 8(0.33) & 2(0.08) & 0.028 \\ \text { Thrombotic events } & 0(0) & 6(0.24) & 0.010\end{array}$

The values are expressed as the mean \pm SD or $n(\%)$ of patients.

BMI, body mass index; AMI, acute myocardial Infarction; ACS, acute coronary syndromes; SBP, Systolic blood pressure; HR: heart rate; HGB, hemoglobin : PLT, platelet count; ADP-PAR: ADP induced platelet aggregation rate; $\mathrm{HbA}_{1 \mathrm{C}}$, hemoglobin A1c; Viscosity, blood viscosity; ALT, alanine aminotransferase; AST, Aspartate aminotransferase; SCr, Serum creatinine; TC, total cholesterol; TG, Triglycerides; eGFR, estimated glomerular filtration rate.

\section{Discussion}

Our study evaluated the ADP-induced Platelet aggregation rate and safety outcomesin patients with the combinedmutations of the CYP2C19, PON1 and $\mathrm{ABCB} 1$ undergoing $\mathrm{PCl}$ receiving clopidogrel and aspirin. Our study demonstrated that patients with multiple mutations of CYP2C19, PON1 and ABCB1 have higher ADPinduced Platelet aggregation rate and more bleeding and thrombotic events. Patients with all mutations of CYP2C19, PON1 and ABCB1 have highest risk of thrombosis and lowest risk of bleeding in patients receiving clopidogrel and aspirin with one-year follow-up.

Multiple studies have consistently shown that PCl-treated patients with impaired clopidogrel-induced platelet inhibition increased the risk for stent thrombosis and ischemic events. Low platelet reactivity is associated with an increased risk of bleeding events, while high platelet reactivity (HPR) is correlated with a high incidence of thrombotic events[12]. Different platelet function testing, such as VerifyNow, Multiplate, vasodilator-stimulated phosphoprotein, thromboelastography (TEG) with platelet mapping and light transmission aggregometry are available for the assessment of on-treatment platelet reactivity to adenosine diphosphate(ADP) $[13,14]$.However, based on the results of platelet function testing, a series of studies were conducted to escalate the appropriate P2Y12 inhibitors on clinical therapy and failed to improve the safety outcomes[15, 16].Peng and his collaborators[3] explored the relationships of the effects of CYP2C19 and PON1 Q192R polymorphism and ADPinduced platelet inhibition by thrombelastography (TEG) in ACS patients with clopidogrel. They found individual CYP2C19 and PON1 variants influenced platelet response on clopidogrel and both LOF CYP2C19 and PON1 192R variants are independent risk factors of high platelet reactivity. However, they didn't evaluate the influence of the multiple variants on safety outcomes. Some researchers measured P2Y12 platelet reaction units using the VerifyNow P2Y12 test to evaluate the effect of clopidogrel on platelet aggregation. Treatment with prasugrel significantly reduced HPR compared with clopidogrelby P2Y12 reaction unitthresholds[17].For patients with longterm DAPT (aspirin + clopidogrel) after PCl with coronary stent implantation, switching from clopidogrel to prasugrel resulted in a stable reduction in PRU, regardless of CYP2C19 polymorphism[18]. In our study, we used ADP-induced platelet aggregation rate to evaluate the platelet response on clopidogrel. After $\mathrm{PCl}$, several established risk factors, such as age, gender, hepatic function, Hemoglobin and genetic polymorphism have been incorporated into a statistical model for clopidogrel response prediction. While these factors could'tbe used to fully explain future bleeding and thrombosis events. In order to exclude the potential bias of other factors, we performed case-control matching procedure and compared the consistency between the genetic polymorphism 
and ADP-induced platelet aggregation rate, we found patients with allelic mutations of CYP2C19, PON1 and ABCB1 have high platelet aggregation rate on clopidogrel.

Besides, we also found patients with combined mutations of CYP2C19, PON1 and ABCB1 have higher risk of thrombosis and lower risk of bleeding in patients receiving clopidogrel and aspirin with one-year follow-up. In a $\mathrm{PCl}$ population taking clopidogrel, the interest in genetic testing for clopidogrel metabolism has grown in recent years to augment standard risk evaluation. The allelic mutant of CYP2C19 which play a significant role in the first oxidation step will lead to lower ADP-induced platelet aggregation rate and higher risk of thrombosis. But the influence on individual PON1 and ABCB1variants iscontradictory and controversial. Chen et al[10] and Hulot et al[19] found the allelic mutant of PON1 was not a major determinant on the clopidogrel pharmacokinetics, pharmacodynamics, and clinical efficacy. While Nishio et al[20],Chen et al[8]and Li et al[21]drawed the inconsistent conclusions that PON1 Q192R genotype influenced clopidogrel responsiveness by relative platelet inhibition, especially in CYP2C19 loss-of-function carriers. Similar disputes also occurred in ABCB1 genotype[2225]. Most studies revealed the individual CYP2C19, PON1 and ABCB1variants on the platelet reactivity and clinical outcomes on patients receiving long-term DAPT (aspirin + clopidogrel) after PCl. A multicomponent guiding approach to personalize clopidogrel treatment is needed[26]. Some studies focus on the combined status of two at-risk variantsin $\mathrm{PCl}$ patients on clopidogrel[3, 8, 9, 20].In the prior studies, both the CYP2C19 poor matebolism status and ABCB1 3435 TT genotype wasconsidered to influence the clopidogrel metabolization and transportationandcontinuously elicited clopidogrel response[5]. The individuals carrying both at-risk variants showed the highest event rate compared to those with other combined genetic subsets. They could not find any association between adverse events and PON-1 genotypes. However,Peng et al found both mutations of CYP2C19 and PON1 192R variants are independent risk factors of high platelet reactivity except ABCB1 variants[3]. There is a paucity of data regarding the combined effect of three at-risk variants, and no data are available to evaluate the incremental prognostic value of their combined effect over conventional clinical risk factors. In our study, we evaluated the combined effect of three at-risk variants on clinical outcomes of patients receiving clopidogrel and aspirin. The lower risk of bleeding and higher risk of thrombosis was observed on interaction among carriers with different number of variants. Besides, regardless of carrying or not carrying CYP2C19 variants, both of PON1 and ABCB1 variants increased the rate of thrombotic events.

There were still some limitations in our study. Firstly, the sample size was relatively small. Secondly, we didn't use another clinically validated and standardized platelet function testing, such as P2Y12 reaction unit (PRU), vasodilator-stimulated phosphoprotein or TEGwith platelet mapping, to define the platelet reactivity in the follow up duration 12 months. Lastly, in this retrospective study, we only included clinical cases with genetic test and platelet aggregation rate and complete follow-up data within one year. This may lead to bias in the inclusion of cases for ignoring patients who transferred to other hospitals and lost to follow-up after adverse events. Finally, we did not exclude the effects of other metabolism enzymes on clopidogrel resistance. Despite we concluded that patients with all mutations of CYP2C19, PON1 and ABCB1 have highest risk of thrombosis and lowest risk of bleeding in patients receiving clopidogrel and aspirin with one-year follow-up duration. Further large-scale and prospective multi-center collaborative research is still essentialto draw definite conclusions on the current issues.

\section{Conclusion}

Multiple genetic polymorphism influence platelet reactivity, bleeding and thrombosis risk to Clopidogrel after percutaneous coronary intervention.

Page $14 / 18$ 


\section{Abbreviations}

PCI,Percutaneous Coronary Intervention;ADP,adenosine diphosphate; BMI, body mass index; AMI, acute myocardial Infarction; ACS, acute coronary syndromes; SBP,Systolic bloodpressure; HR heart rate;HGB,hemoglobin:PLT, platelet count;ADP-PAR:ADP induced platelet aggregation rate; $\mathrm{HbA}_{1 \mathrm{C}}$, hemoglobin A1c; Viscosity, blood viscosity; ALT, alanineaminotransferase; AST, Aspartate aminotransferase; SCr, Serum creatinine; TC, total cholesterol; TG, Triglycerides; eGFR, estimated glomerular filtration rate; $C$, ABCB1 3435C > T wild allele; CT, ABCB1 3435C > T heterozygotic allele; TT, ABCB1 3435C > T homozygotic allele. GG, PON1 575A > G wild allele; GA:PON1 575A > G heterozygotic allele; AA, PON1 575A > G homozygotic allele.CYP2C19(No), CYP2C19*1*1;CYP2C19(Mu), CYP2C19*1*2 or *1*3 or *2*2 or *3*3; ABCB1(No), ABCB1 CC, ABCB1 3435C > T wild allele; ABCB1(Mu),ABCB1CT/TT, ABCB1 3435C > T heterozygotic or homozygotic allele; PON1(No), PON1GG, PON1 575A > G wild allele; PON1(Mu), PON1GA/AA, PON1 575A > G heterozygotic or homozygotic allele.

\section{Declarations}

\section{Ethics approval and consent to participate}

The protocol used was approved by the Ethics Committee of The Fifth people's Hospital of Shanghai, Fudan University (No. 2018-192).Due to the retrospective nature of the study, informed consent was waived by the Ethics Committee of The Fifth people's Hospital of Shanghai. All the human data have been performed in accordance with the declaration of Helsinki.All methods were carried out in accordance with the declaration of Helsinki and relevant guidelines and regulations.

\section{Consent for publication}

All the authors are consent for publication.

\section{Availability of data and material}

The datasets used and/or analysed during the current study available from the corresponding author on reasonable request.

\section{Conflicts of interests}

All the authors have no conflict of interest to declare.

\section{Funding}

The study was supported by grants from Shanghai Minhang Health and Family Planning Commission (2018MW03), Shanghai Municipal Health and Family Planning Commission (20194Y0258) and The Fifth People's Hospital of Shanghai (2018WYZT04). 


\section{Author contributions}

Yan Yu and Hui Jin concepted and designed this study. Patients follow-up and Data extraction was carried out by Xiaoying Shen and Hui Jin. All statistical analysis was performed by Jinfei Song and was double checked by HuiJin. This manuscript was drafted by Yu Yan, Qing Liang and Guangchun Sun are critically reviewed for improving overall quality.

\section{Acknowledgments}

Not applicable.

\section{References}

1. So HS, So MG, Kang SI, Park JI, Lee JH, Kim U, and Park JS. Long-Term Safety and Efficacy of Extended Dual Antiplatelet Therapy After Drug-Eluting Stent Implantation in Real-World Practice. Circ J. 2020; 84(12):2175-2184.

2. Klein MD, Williams AK, Lee CR, and Stouffer GA. Clinical Utility of CYP2C19 Genotyping to Guide Antiplatelet Therapy in Patients With an Acute Coronary Syndrome or Undergoing Percutaneous Coronary Intervention. Arterioscler Thromb Vasc Biol. 2019; 39(4):647-652.

3. Peng W, Shi X, Xu X, and Lin Y. Both CYP2C19 and PON1 Q192R Genotypes Influence Platelet Response to Clopidogrel by Thrombelastography in Patients with Acute Coronary Syndrome. Cardiovasc Ther. 2019; 2019:3470145.

4. Mega JL, Close SL, Wiviott SD, Man M, Duvvuru S, Walker JR, Sundseth SS, Collet JP, Delaney JT, Hulot JS, et al. PON1 Q192R genetic variant and response to clopidogrel and prasugrel: pharmacokinetics, pharmacodynamics, and a meta-analysis of clinical outcomes. J Thromb Thrombolysis. 2016; 41(3):37483.

5. Park MW, Her SH, Kim CJ, SunCho J, Park GM, Kim TS, Choi YS, Park CS, Koh YS, Park HJ, et al. Evaluation of the incremental prognostic value of the combination of CYP2C19 poor metabolizer status and ABCB1 3435 TT polymorphism over conventional risk factors for cardiovascular events after drug-eluting stent implantation in East Asians. Genet Med. 2016; 18(8):833-41.

6. Calderon-Cruz B, Rodriguez-Galvan K, Manzo-Francisco LA, Vargas-Alarcon G, Fragoso JM, Pena-Duque MA, Reyes-Gomez CA, Martinez-Rios MA, and De la Pena-Diaz A. C3435T polymorphism of the ABCB1 gene is associated with poor clopidogrel responsiveness in a Mexican population undergoing percutaneous coronary intervention. Thromb Res. 2015; 136(5):894-8.

7. Liu X, Luo Y, Lai Y, Yao Y, Li J, Wang Y, Zheng SL, Xu J, and Liu X. Effect of genetic and coexisting polymorphisms on platelet response to clopidogrel in Chinese Han patients with acute coronary syndrome. J Genet. 2016; 95(2):231-7.

8. Chen Y, Huang X, Tang Y, Xie Y, and Zhang Y. Both PON1 Q192R and CYP2C19*2 influence platelet response to clopidogrel and ischemic events in Chinese patients undergoing percutaneous coronary intervention. Int $J$ Clin Exp Med. 2015; 8(6):9266-74. 
9. Tang XF, Wang J, Zhang JH, Meng XM, Xu B, Qiao SB, Wu YJ, Chen J, Wu Y, Chen JL, et al. Effect of the CYP2C19 2 and 3 genotypes, ABCB1 C3435T and PON1 Q192R alleles on the pharmacodynamics and adverse clinical events of clopidogrel in Chinese people after percutaneous coronary intervention. Eur J Clin Pharmacol. 2013; 69(5):1103-12.

10. Chen DY, Wang CY, Wen MS, Lee TH, Chu Y, Hsieh MJ, Chang SH, Lee CH, Wang JL, Chen CC, et al. Paraoxonase-1 is not a major determinant of stent thrombosis in a Taiwanese population. PLoS One. 2012; 7(6):e39178.

11. Meschia JF, Walton RL, Farrugia LP, Ross OA, Elm JJ, Farrant M, Meurer WJ, Lindblad AS, Barsan W, Ching M, et al. Efficacy of Clopidogrel for Prevention of Stroke Based on CYP2C19 Allele Status in the POINT Trial. Stroke. 2020; 51(7):2058-2065.

12. Tantry US, Bonello L, Aradi D, Price MJ, Jeong YH, Angiolillo DJ, Stone GW, Curzen N, Geisler T, Ten Berg J, et al. Consensus and update on the definition of on-treatment platelet reactivity to adenosine diphosphate associated with ischemia and bleeding. J Am Coll Cardiol. 2013; 62(24):2261-73.

13. Sibbing D, Aradi D, Alexopoulos D, Ten Berg J, Bhatt DL, Bonello L, Collet JP, Cuisset T, Franchi F, Gross L, et al. Updated Expert Consensus Statement on Platelet Function and Genetic Testing for Guiding P2Y12 Receptor Inhibitor Treatment in Percutaneous Coronary Intervention. JACC Cardiovasc Interv. 2019; 12(16):1521-1537.

14. Aradi D, Storey RF, Komocsi A, Trenk D, Gulba D, Kiss RG, Husted S, Bonello L, Sibbing D, Collet JP, et al. Expert position paper on the role of platelet function testing in patients undergoing percutaneous coronary intervention. Eur Heart J. 2014; 35(4):209-15.

15. Trenk D, Stone GW, Gawaz M, Kastrati A, Angiolillo DJ, Muller U, Richardt G, Jakubowski JA, and Neumann FJ. A randomized trial of prasugrel versus clopidogrel in patients with high platelet reactivity on clopidogrel after elective percutaneous coronary intervention with implantation of drug-eluting stents: results of the TRIGGER-PCI (Testing Platelet Reactivity In Patients Undergoing Elective Stent Placement on Clopidogrel to Guide Alternative Therapy With Prasugrel) study. J Am Coll Cardiol. 2012; 59(24):2159-64.

16. Collet JP, Cuisset T, Range G, Cayla G, Elhadad S, Pouillot C, Henry P, Motreff P, Carrie D, Boueri Z, et al. Bedside monitoring to adjust antiplatelet therapy for coronary stenting. N Engl J Med. 2012; 367(22):21009.

17. So DY, Wells GA, McPherson R, Labinaz M, Le May MR, Glover C, Dick AJ, Froeschl M, Marquis JF, Gollob MH, et al. A prospective randomized evaluation of a pharmacogenomic approach to antiplatelet therapy among patients with ST-elevation myocardial infarction: the RAPID STEMI study. Pharmacogenomics J. 2016; 16(1):71-8.

18. Shimamatsu J, Sasaki KI, Katsuki Y, Kawasaki T, Murasato Y, Ajisaka H, Yokoi H, Tashiro H, Harada A, Hirakawa $Y$, et al. Prasugrel effectively reduces the platelet reactivity units in patients with genetically metabolic dysfunction of cytochrome P450 2C19 who are treated with long-term dual antiplatelet therapy after undergoing drug-eluting stent implantation. Heart Vessels. 2020; 35(3):312-322.

19. Hulot JS, Collet JP, Cayla G, Silvain J, Allanic F, Bellemain-Appaix A, Scott SA, and Montalescot G. CYP2C19 but not PON1 genetic variants influence clopidogrel pharmacokinetics, pharmacodynamics, and clinical efficacy in post-myocardial infarction patients. Circ Cardiovasc Interv. 2011; 4(5):422-8.

20. Nishio R, Shinke T, Otake H, Nakagawa M, Inoue T, Hariki H, Osue T, Taniguchi Y, Iwasaki M, Hiranuma N, et al. Paraoxonase-1 activity affects the clopidogrel response in CYP2C19 loss-of-function carriers. Thromb

Page 17/18 
Res. 2013; 132(5):558-64.

21. Li X, Zhang L, Chen X, Qu F, Li J, Ma C, Yang J, Xu B, Wang H, Xu Q, et al. PON1 Q192R genotype influences clopidogrel responsiveness by relative platelet inhibition instead of on-treatment platelet reactivity. Thromb Res. 2013; 132(4):444-9.

22. Biswas M, Rahaman S, Biswas TK, and Ibrahim B. Effects of the ABCB1 C3435T single nucleotide polymorphism on major adverse cardiovascular events in acute coronary syndrome or coronary artery disease patients undergoing percutaneous coronary intervention and treated with clopidogrel: A systematic review and meta-analysis. Expert Opin Drug Saf. 2020; 19(12):1605-1616.

23. Hou X, Han W, Gan Q, Liu Y, and Fang W. CYP2C19 and ABCB1 genetic polymorphisms correlate with the recurrence of ischemic cardiovascular adverse events after clopidogrel treatment. J Clin Lab Anal. 2018; 32(5):e22369.

24. Namazi S, Sahebi E, Azarpira N, Rostami-Yalmeh J, Kojuri J, and Khalili A. Association of ABCB1 Gene Polymorphisms and Clopidogrel Responsiveness in Iranian Patients undergoing Percutaneous Coronary Intervention. Iran J Pharm Res. 2020; 19(2):307-316.

25. Zhang JH, Tang XF, Zhang Y, Wang J, Yao Y, Ma YL, Xu B, Gao RL, Gao Z, Chen J, et al. Relationship between ABCB1 polymorphisms, thromboelastography and risk of bleeding events in clopidogrel-treated patients with ST-elevation myocardial infarction. Thromb Res. 2014; 134(5):970-5.

26. Valeria C, Carmine S, Valentina M, Teresa I, Maria C, Martina T, Giancarlo A, Giovanna N, Graziamaria C, and Amelia $\mathrm{F}$. The need of a multicomponent guiding approach to personalize clopidogrel treatment. Pharmacogenomics J. 2021; 21(2):116-127. 